\title{
Erratum to: Relationship between Clinical, MRI, and Arthroscopic Findings: A Guide to Correct Diagnosis of Meniscal Tears
}
P. Antinolfi ${ }^{1}$
R. Cristiani ${ }^{2}$
F. Manfreda ${ }^{2}$
S. Bruè ${ }^{3}$
V. Sarakatsianos ${ }^{4}$
G. Placella ${ }^{5}$
M. Bartoli ${ }^{5}$
A. Caraffa ${ }^{1,2}$

${ }^{1}$ Division of Orthopedics and Trauma Surgery, S. Maria della Misericordia Hospital, Perugia, Italy

2 Department of Orthopedics and Traumatology, University of Perugia, Italy

${ }^{3}$ International Orthopedics and Traumatology Institute, Arezzo, Italy

${ }^{4}$ Capio Artro Clinic/Stockholm Sports Trauma Research Center,

Stockholm, Sweden

${ }^{5}$ Department of Othopaedics, U. Cattolica del Sacro Cuore, Gemelli

Ospital, Rome, Italy

Joints 2018;6:71-72.

\section{ERRATUM}

It has been brought to the publisher's attention that the name of author R. Cristiani was published incorrectly in the above article in the journal Joints, Volume 5, Number 3, 2017 (DOI: 10.1055/s-0037-1605583). The author's name was originally published as "R. Crisitiani." The correct listing of the author's name appears above. 